\title{
Model-Based Fault Detection and Estimation for Linear Time Invariant and Piecewise Affine Systems by Using Quadratic Boundedness
}

\author{
Angelo Alessandri, Francesca Boem, and Thomas Parisini
}

\begin{abstract}
Quadratic boundedness is a notion of stability that is adopted to investigate the design of observers for dynamic systems subject to bounded disturbances. We will show how to exploit such observers for the purpose of fault detection. Toward this end, first of all we present the naive application of quadratic boundedness to construct state observers for linear time-invariant systems with state augmentation, i.e., where additional variables may be introduced to account for the occurrence of a fault. Then a Luenberger observer is designed to estimate the augmented state variable of the system in such a way to detect the fault by using a convenient threshold selection. Finally, such an approach is extended to piecewise affine systems by presenting a hybrid Luenberger observer and its related design based on quadratic boundedness. The design of all the observers for both linear time-invariant and piecewise affine systems can be done by using linear matrix inequalities. Simulation results are provided to show the effectiveness of the proposed approach.
\end{abstract}

\section{INTRODUCTION}

Reliability is a key requirement for modern systems. Therefore, fault diagnosis is a research field that has been in the front end of the technological evolution for a few decades and has attracted the attention from the research and industrial community, as testified by many important survey papers and books (see [1]-[3] just as examples). Recent effort has been directed at investigating solutions for monitoring distributed, large-scale and interconnected systems [4]-[11].

When dealing with model-based approaches [2], due to the presence of uncertainties, one of the main issues is the definition of thresholds for some residual signals defined to be sensitive to the presence of faults. Different solutions have been proposed, either considering deterministic bounds on the uncertainties so to guarantee the absence of false alarms [12], or a stochastic characterization of noises and disturbances in order to set bounds on the allowed falsealarms rate [11]. A major challenge is represented by the fact that, in order to be robust to the noises, thresholds are often conservative, thus leading to scenarios where the uncertainties may hide the presence of faults.

This work has been partially supported by European Union's Horizon 2020 research and innovation programme under grant agreement No 739551 (KIOS CoE).

A. Alessandri is with the University of Genoa (DIME), Italy. Email: alessandri@dime.unige.it

F. Boem is with the Department of Electronic and Electrical Engineering at University College London, UK. Email: francesca.1.boem@gmail.com

T. Parisini is with the Department of Electrical and Electronic Engineering at the Imperial College London, UK, with the KIOS Research and Innovation Centre of Excellence, University of Cyprus, and also with the Department of Engineering and Architecture at University of Trieste, Italy. Email: t.parisini@gmail.com
In this paper, we propose a new design method for the application of state estimation to the fault detection problem. The proposed method is based on positive invariance and quadratic boundedness (QB) [13]. Positive invariant sets have already been exploited in the literature for the purpose of fault detection and isolation (see, for example, [14]-[17]). QB allows to deal with positively invariant sets in quite an intuitive way and it provides upper bounds on the trajectories of the state of a system subject to bounded disturbances, which may be fruitfully exploited for the purpose of fault diagnosis. Here we present some preliminary results on how the QB properties can be adopted as a guidance for the selection of suitable fault detection thresholds. Other applications of QB are reported in the literature for both output feedback control [18] and state estimation [19], [20]. No application of QB to fault diagnosis is known to the authors.

Based on the availability of information on the plant and practical experience on the possible malfunctions, one can develop a model with additional state variables that can take into account the occurrence of faults. The idea of enriching the system model with new variables is pretty popular in adaptive control and it is referred to as "state augmentation." For the purpose of fault detection, it can be useful as well to monitor the system by estimating all the state variables and especially those describing malfunctions such as sensor bias or actuator block [21]. Estimation can be accomplished by means of Luenberger observers and, if the modeling framework is extended from linear time-invariant (LTI) to piecewise affine (PWA) systems, by using hybrid observers that account for the specific mode denoting the subset of the state space in which the state trajectory lays. The importance for methods aimed at performing estimation for PWA and switched systems is motivated also by some recent results concerning especially fault diagnosis [22]-[24].

There exists a vast literature on hybrid and switching systems but very little can be found in estimation. The first results are reported in [25]-[27] but, as pointed out in [28], the problem to design state observers for such systems is somehow still open because of the difficulty to deal with the observability of the mode under realistic conditions. Thus, we propose also an extension of the QB approach to fault estimation for PWA systems by using a prediction of the system mode and proving the boundedness of the estimation error. Likewise for LTI systems, the design of the fault estimators can be accomplished by using linear matrix inequalities (LMIs), which allow for an effective design thanks to well-established semi-definite programming (SDP) 
tools [29].

The paper is organized as follows. In Section II, we present the naive idea of applying QB to state-augmented LTI systems for the purpose of fault diagnosis. The construction of state observers to perform this task is described in Section III, while Section IV is focused on a convenient design procedure based on LMIs. Section V deals with the extension of the proposed approach to PWA systems. Simulations results are reported in Section VI. Finally, the conclusions are drawn in Section VII.

Let $(x, y):=\left[x^{\top}, y^{\top}\right]^{\top}$, where $x$ and $y$ are column vectors. The minimum and maximum eigenvalues of a real, symmetric matrix $P$ are denoted by $\lambda_{\min }(P)$ and $\lambda_{\max }(P)$, respectively. Moreover, $P>0(P<0)$ means that it is also positive (negative) definite. Given a generic matrix $M,|M|:=\left(\lambda_{\max }\left(M^{\top} M\right)\right)^{1 / 2}=\left(\lambda_{\max }\left(M M^{\top}\right)\right)^{1 / 2}$ and hence, for a vector $v,|v|:=\left(v^{\top} v\right)^{1 / 2}$ is its Euclidean norm.

\section{Problem Formulation}

Let us consider the dynamic system

$$
\begin{aligned}
\dot{x} & =A x+B u+D w \\
y & =C x+E w
\end{aligned}
$$

where $x \in \mathbb{R}^{n}$ is the state, $u \in \mathbb{R}^{p}$ is the control input, $y \in \mathbb{R}^{m}$ is the output; $w \in \mathbb{R}^{q}$ is a vector that collects all the noises that may affect both dynamics and measurements. Without loss of generality, we assume that such noises are bounded as follows.

Assumption 1: The disturbance $t \mapsto w(t) \in \mathbb{R}^{q}$ is such that $\left|w_{i}(t)\right| \leq 1, i=1, \ldots, r$ for all $t \geq 0$.

For the sake of simplicity, in this first part of the paper, we assume that system (1) accounts for both the dynamics of the process to monitor and additional variables that model the occurrence of faults. This can be obtained by augmenting the state vector with some variables describing the fault dynamics. With this formulation we can model both process and sensors ' bias faults as well as additive faults with known linear dynamical structure. In the following we give an example.

Example 1: Consider a SISO plant described by

$$
\begin{aligned}
& \dot{\xi}=\bar{A} \xi+\bar{B} u \\
& y=\bar{C} \xi
\end{aligned}
$$

where $\xi \in \mathbb{R}^{n}, u \in \mathbb{R}$, and $y \in \mathbb{R}$. If such a system is subject to sensor bias faults [30] so that the output equation becomes $y=\bar{C} \xi+\theta$, where $\theta(t) \in \mathbb{R}$ can be considered an additional state variable with a simple augmented dynamics given by $\dot{\theta}=0$. Thus, one may refer to system (1) with $x=(\xi, \theta) \in \mathbb{R}^{n+1}$ and

$$
A=\left(\begin{array}{cc}
\bar{A} & 0_{n} \\
0_{n}^{\top} & 0
\end{array}\right) \quad B=\left(\begin{array}{c}
\bar{B} \\
0
\end{array}\right) \quad C=\left(\begin{array}{ll}
\bar{C} & 1
\end{array}\right) .
$$

where $0_{n}$ is a column vector made of $n$ zeros. Note also that the pair $(A, C)$ is observable if $(\bar{A}, \bar{C})$ is observable.
In the following, we will present an approach to fault detection based on the idea to estimate the state of (1), which includes state variables belonging to the original model of the plant and additional variables introduced to account for faults. The decision about the occurrence of a fault may be taken by comparing the estimate of such additional variables with their nominal values. In the case of Example 1, one can detect the presence of a sensor bias fault by analyzing the estimate of $\theta$. Clearly, it is crucial to design a convenient observer together with a reliable decision scheme by taking into account the information available on the disturbances. Quadratic boundedness turns out to be well-suited to dealing with this problem. A bound on the estimation error will be properly found out, by exploiting the notion of quadratic boundedness. This property can be used for the selection of less conservative thresholds for fault detection, by accepting the possible presence of false alarms.

\section{ESTIMATION FOR LTI SYSTEMS AND QUADRATIC BOUNDEDNESS}

A simple Luenberger observer for (1) is described by

$$
\dot{\hat{x}}=A \hat{x}+B u+L(y-C \hat{x})
$$

where $\hat{x}(t) \in \mathbb{R}^{n}$ is the estimate of $x(t)$ and $L \in \mathbb{R}^{n \times m}$ is the observer gain to be chosen. Thus, we need to assume the following.

Assumption 2: The pair $(A, C)$ is detectable.

The assumption above allows one to construct an observer with an asymptotically stable dynamics of the estimation error $e(t):=x(t)-\hat{x}(t) \in \mathbb{R}^{n}$ in a noise-free setting. However, in the presence of disturbances, it is important to reject their effect in general and, in our specific context, to devise a decision scheme able to detect faults or malfunctions.

Concerning the state-augmented framework, the estimation error after the transient keeps staying in an invariant set in the absence of fault, and the bigger the noises, the larger such a set. Among the various choices, we will rely on ellipsoidal invariant sets related to quadratic boundedness [13], [18]-[20].

Using (1) and (2), the dynamics of the estimation error reads

$$
\dot{e}=(A-L C) e+(D-L E) w
$$

Let define quadratic boundedness as follows [13].

Definition 1: The estimation error is said to be quadratically bounded with Lyapunov matrix $P>0$ if

$$
\begin{aligned}
e^{\top} P e>1 & \Rightarrow 2 e^{\top} P((A-L C) e \\
& +(D-L E) w)<0, \forall w \in[-1,1]^{q} .
\end{aligned}
$$

Owing to (4), the set $\mathcal{E}_{\mathrm{p}}:=\left\{e \in \mathbb{R}^{n}: e^{\top} P e \leq 1\right\}$ turns out to be positively invariant, it contains the reachable set from the origin, and it is attractive (i.e., if the error is out of $\mathcal{E}_{\mathrm{p}}$, it approaches $\mathcal{E}_{\mathrm{p}}$ asymptotically). Moreover, the error is upper bounded as follows:

$$
|e(t)|^{2} \leq \frac{1}{\lambda_{\min }(P)} \max \left\{e(0)^{\top} P e(0), 1\right\}
$$


for all $t \geq 0$. Clearly, such a bound combines the transient and steady conditions. After the transient, we have

$$
|e(t)| \leq 1 / \sqrt{\lambda_{\min }(P)} .
$$

Based on the aforesaid, we can state the following.

Theorem 1: The estimation error is quadratically bounded if and only if there exist $P>0, Y \in \mathbb{R}^{n \times m}, \alpha \in \mathbb{R}_{>0}^{q}$, and $\beta>0$ such that

$$
\begin{aligned}
& \left(\begin{array}{cc}
A^{\top} P-C^{\top} Y^{\top}+P A-Y C+\beta P & P D-Y E \\
\star & -\operatorname{diag}(\alpha)
\end{array}\right)<0 \\
& \sum_{i=1}^{q} \alpha_{i}-\beta \leq 0
\end{aligned}
$$

with $L=P^{-1} Y$.

Proof: Let $V(e):=e^{\top} P e$ a Lyapunov function. Clearly, the condition of quadratic boundedness can be reformulated as follows

$$
V(e)>1 \Rightarrow \frac{\mathrm{d} V(e)}{\mathrm{d} t}<0
$$

for all $w_{i} \in \mathbb{R}$ s.t. $w_{i}^{2} \leq 1$. Moreover, since

$$
\begin{aligned}
& \frac{\mathrm{d} V(e)}{\mathrm{d} t}=e^{\top}\left[(A-L C)^{\top} P+P(A-L C)\right] e \\
& +w^{\top}\left(D^{\top}-E^{\top} L^{\top}\right) P e+e^{\top} P(D-L E) w<0
\end{aligned}
$$

and $-e^{\top} P e+1<0$, using [29, S-procedure, p. 23] it follows that there exist $\alpha_{i}>0$ and $\beta>0$ s.t.

$$
\begin{aligned}
& e^{\top}\left[(A-L C)^{\top} P+P(A-L C)\right] e+w^{\top}\left(D^{\top}\right. \\
& \left.-E^{\top} L^{\top}\right) P e+e^{\top} P(D-L E) w+e^{\top} \beta P e-\sum_{i=1}^{q} \alpha_{i} w_{i}^{2} \\
& +\sum_{i=1}^{q} \alpha_{i}-\beta \leq 0
\end{aligned}
$$

and hence, using some well-known LMI technicality with $Y=P L$, we get (7a) and (7b).

The proof of Theorem 1 shows that (7a) can be satisfied if and only if $A-L C$ is Hurwitz.

\section{FAUlT DETECTION LOGIC AND THRESHOLDING}

Fault detection may be accomplished by analyzing the estimated state variables and especially the additional variables, introduced to model the fault. The steady-state bound (6) can be used for estimator design and both threshold selection.

To illustrate the motivation, consider Example 1 with the last state variable accounting for the bias. Thus, in faultfree conditions at steady state, the error associated with the estimate of the bias with respect to its nominal value is not larger than $1 / \sqrt{\lambda_{\min }(P)}$. Thus, a sufficiently high variation of the bias may be estimated by the observer and detected by the derived bound, that is, if in steady-state

$$
|e(t)|>1 / \sqrt{\lambda_{\min }(P)},
$$

then we have fault detection without false alarms.

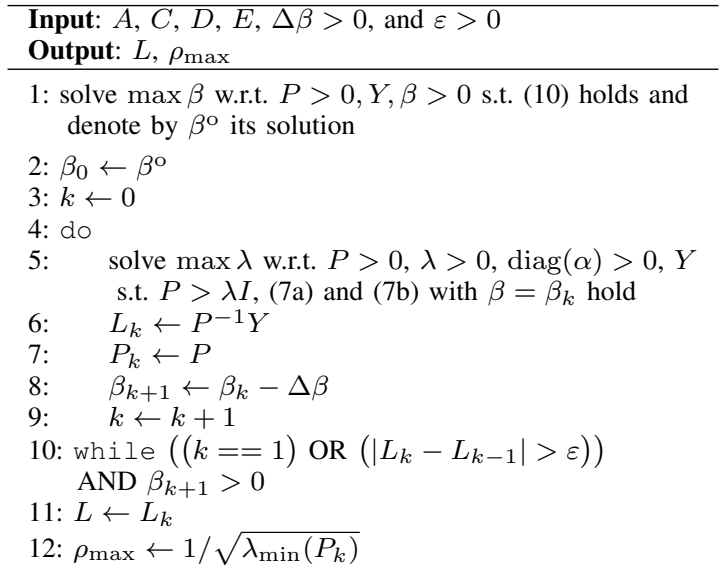

TABLE I

DESIGN PROCEDURE

Owing to uncertainty the selection of a too tight detection threshold may cause false alarms, whereas, in the case the threshold is too large, this may lead to fault misdetection. The bound in (8) guarantees the absence of false alarms in steady state but may be conservative. Since this bound depends on the choice of the matrix $P$, the goal of the design may consist in maximizing $\lambda_{\min }(P)$ in order to reduce it. This can be obtained by maximizing $\lambda$ subject to the LMI $P>\lambda I$ as follows:

$$
\begin{aligned}
\max _{P, Y, \alpha, \beta} \lambda & \\
\text { s.t. } & P>0, \lambda>0, \operatorname{diag}(\alpha)>0, \beta>0, \\
& P>\lambda I,(7 \mathrm{a}), \text { and (7b) hold. }
\end{aligned}
$$

Such a problem is not in an LMI form that can be solved by using standard SDP tools. Toward this end, note that the stability conditions require the satisfaction of the inequality

$$
A^{\top} P-C^{\top} Y^{\top}+P A-Y C+\beta P<0
$$

if $w=0$. Such a condition is not an LMI in $P, Y$, and $\beta$; however, one can solve a generalized eigenvalue (GEV) problem by maximizing $\beta$ to ensure a transient as fast as possible [29]. Based on the solution of such a problem, we may take the resulting maximum $\beta$ as a starting upper bound to be reduced so as to get the satisfaction of (7) while maximizing $\lambda_{\min }(P)$. The proposed design approach is summarized in Table I, where $\varepsilon>0$ is the admitted tolerance and the gain $L$ is the final result of the procedure together with the steady-state $\mathrm{QB}$ bounds $\rho_{\max }$.

Remark 1: It is important to note that the proposed approach may be useful to design residual generators even in case a model of the fault is not available. Based on the nominal model, we can define the residual signal $r(t):=y(t)-$ $C \hat{x}(t)$ and, by noting that $r(t)=C e(t)+E w(t)$, at each time step we compare such a residual with a suitable fault detection threshold, where the fault-free condition is

$$
\begin{aligned}
|r(t)| & \leq|C e(t)|+|E w(t)| \leq|C| / \sqrt{\lambda_{\min }(P)}+|E||w| \\
& \leq|C| / \sqrt{\lambda_{\min }(P)}+q|E|=: r_{\max }
\end{aligned}
$$


Thus, in case $|r(t)|>r_{\max }$, we can detect the fault guaranteeing the absence of false alarms. Moreover, the goal of maximizing $\lambda_{\min }(P)$ according to (9) allows to get a threshold less conservative as much as possible.

Remark 2: The proposed QB bound $\rho_{\max }$, designed using the procedure in Table I, represents the least conservative threshold in the form of (6) able to guarantee the absence of false alarms in steady state. In some real-world applications, it may be anyway conservative, but it can be used as a guidance for the definition of suitable less conservative fault detection thresholds at the cost of accepting the possible presence of false alarms.

\section{EXTENSION OF FAULT Estimation to PWA SYSTEMS}

In this section, we will address the same problem of Section II for a class of PWA systems described by

$$
\begin{aligned}
& \dot{x}=A_{\sigma} x+B u+D w \\
& y=C_{\sigma} x+E w \\
& \sigma\left(t^{+}\right)=F\left(x\left(t^{-}\right), u\left(t^{-}\right)\right)
\end{aligned}
$$

where $u \in U \subset \mathbb{R}^{p}$ bounded and $\sigma \in \Sigma:=\{1, \ldots, s\}$ represents a discrete state, which will be denoted as "mode" of the system. The matrices $A_{i}$ and $C_{i}, i \in \Sigma$, are known as well as the impulsive mapping $(x, t) \mapsto F(x, u) \in \Sigma$ but, since only $y(t)$ is available though subject to measurement noises and the continuous and discrete states are unknown at any $t \geq 0$ in general.

The PWA (12) allows to extend the applicability of the proposed approach in such a way to deal with a more general class of faults that may change the system dynamics. In practice, we implicitly assume to know anyway all the possible nominal and faulty dynamics.

Assumption 3: There exists a bounded set $X \subset \mathbb{R}^{n}$ s.t. $x(t) \in X$ for all $t \geq 0, u \in U$, and $w \in[-1,1]^{q}$.

To perform estimation for (12), we use the following hybrid Luenberger observer:

$$
\begin{aligned}
& \dot{\hat{x}}=A_{\hat{\sigma}} \hat{x}+B u+L_{\hat{\sigma}}\left(y-C_{\hat{\sigma}} \hat{x}\right) \\
& \hat{\sigma}\left(t^{+}\right)=F\left(\hat{x}\left(t^{-}\right), u\left(t^{-}\right)\right)
\end{aligned}
$$

where $\hat{x}(t) \in \mathbb{R}^{n}$ is the state estimate of $x(t)$ at any $t \geq 0$. The mode is predicted according to the impulsive law (13b). The gain matrices $L_{i}$ associated with each estimated mode can be determined by applying the same approach of Section II. More specifically, the following theorem holds.

Theorem 2: If there exist $P>0$ and $Y_{i} \in \mathbb{R}^{n \times m}$ for $i=1, \ldots, s, \alpha \in \mathbb{R}_{>0}^{q}$, and $\beta>0$ such that

$$
\begin{gathered}
\left(\begin{array}{c}
A_{i}^{\top} P-C_{i}^{\top} Y_{i}^{\top}+P A_{i}-Y_{i} C_{i}+\beta P \\
\star
\end{array} \quad \begin{array}{c}
P D-Y_{i} E \\
-\operatorname{diag}(\alpha)
\end{array}\right)<0 \\
\sum_{i=1}^{q} \alpha_{i}-\beta \leq 0
\end{gathered}
$$

with $L_{i}=P^{-1} Y_{i}, i=1, \ldots, s$, the estimation error is bounded.

Proof: Let us decompose the estimation error as follows

$$
e=x-\hat{x}=\underbrace{x-\hat{x}_{\mathrm{p}}}_{e_{\mathrm{p}}}+\underbrace{\hat{x}_{\mathrm{p}}-\hat{x}}_{\hat{e}_{\mathrm{p}}}
$$

where $\hat{x}_{\mathrm{p}}(t) \in \mathbb{R}^{n}$ is the state of the "perfect" Luenberger observer, namely an observer based on the instantaneous knowledge of the mode $\sigma(t)$. More specifically, such an observer is given by

$$
\dot{\hat{x}}_{\mathrm{p}}=A_{\sigma} \hat{x}_{\mathrm{p}}+B u+L_{\sigma}\left(y-C_{\sigma} \hat{x}\right)
$$

and provides an error $e_{\mathrm{p}}(t)$ that is quadratically bounded owing to (14). As a consequence, using Assumption 3 it follows that also $\hat{x}_{\mathrm{p}}(t)$ is bounded. Since we can easily find that

$$
\begin{aligned}
\dot{\hat{e}}_{\mathrm{p}} & =\left(A_{\hat{\sigma}}-L_{\hat{\sigma}} C_{\hat{\sigma}}\right) \hat{e}_{\mathrm{p}}+\left(A_{\sigma}-A_{\hat{\sigma}}+L_{\hat{\sigma}} C_{\hat{\sigma}}-L_{\sigma} C_{\sigma}\right) \hat{x}_{\mathrm{p}} \\
& +\left(L_{\sigma}-L_{\hat{\sigma}}\right)\left(C_{\sigma} x+E w\right),
\end{aligned}
$$

it is straightforward to conclude about the boundedness of $e_{\mathrm{p}}(t)$ and hence of $e(t)$.

Note that the pairs $\left(A_{i}, C_{i}\right), i=1, \ldots, s$, must be detectable to admit the existence of some solution to (14a).

\section{Simulation Results}

For the sake of space limitation, only one case study concerning a PWA autonomous system will be presented. It is inspired by the simple model presented in [31] about two cascaded interconnected systems subject to mutual functional dependencies. Such a model is an autonomous PWA system with $\Sigma=\{1,2,3\}$, where the first mode corresponds to a "safe" plant, while an anomalous behavior occurs in the other two discrete states. Specifically, we have chosen

$$
\begin{aligned}
& A_{1}=\left(\begin{array}{rrrr}
-0.5 & 0 & 1 & 0 \\
0 & -0.5 & 0 & 1 \\
0 & 0 & 0 & 0 \\
0 & 0 & 0 & 0
\end{array}\right) A_{2}=\left(\begin{array}{rrrr}
-0.5 & 0 & 1 & 0 \\
0 & -1 & 0 & 1 \\
0 & 0 & 0 & 0 \\
0 & 0 & 0 & 0
\end{array}\right) \\
& A_{3}=\left(\begin{array}{rrrr}
-1 & 0 & 1 & 0 \\
0 & -0.5 & 0 & 1 \\
0 & 0 & 0 & 0 \\
0 & 0 & 0 & 0
\end{array}\right) \\
& C_{1}=C_{2}=C_{3}=\left(\begin{array}{llll}
1 & 0 & 0 & 0 \\
0 & 1 & 0 & 0
\end{array}\right) \\
& D=\left(\begin{array}{rrrr}
0 & 0 & 0.1 & 0 \\
0 & 0 & 0 & 0.1 \\
0 & 0 & 0 & 0 \\
0 & 0 & 0 & 0
\end{array}\right) E=\left(\begin{array}{rrrr}
0.01 & 0 & 0 & 0 \\
0 & 0.01 & 0 & 0
\end{array}\right)
\end{aligned}
$$

and

$$
F(x, u)= \begin{cases}1 & \text { if } x_{1} \leq \bar{\sigma} \text { and } x_{2} \leq \bar{\sigma} \\ 2 & \text { if } x_{1} \leq \bar{\sigma} \text { and } x_{2}>\bar{\sigma} \\ 3 & \text { if } x_{1}>\bar{\sigma} \text { and } x_{2} \leq \bar{\sigma}\end{cases}
$$

with $\bar{\sigma}=0.4$. 
The design of the observer (13) has been accomplished by using the design procedure in Table I. We have obtained the following results:

$$
\begin{aligned}
& P=\left(\begin{array}{rrrr}
94.1508 & 0 & -20.9304 & 0 \\
0 & 94.1508 & 0 & -20.9304 \\
-20.9304 & 0 & 40.5391 & 0 \\
0 & -20.9304 & 0 & 40.5391
\end{array}\right) \\
& L_{1}=\left(\begin{array}{rr}
3.6486 & 0 \\
0 & 3.6486 \\
2.1926 & 0 \\
0 & 2.1926
\end{array}\right) \quad L_{2}=\left(\begin{array}{rr}
2.7647 & 0 \\
0 & 4.4939 \\
1.74 & 0 \\
0 & 2.1946
\end{array}\right) \\
& L_{3}=\left(\begin{array}{rr}
4.4939 & 0 \\
0 & 2.7647 \\
2.1946 & 0 \\
0 & 1.74
\end{array}\right) \alpha=\left(\begin{array}{l}
0.0404 \\
0.0404 \\
0.2026 \\
0.2026
\end{array}\right) \beta=0.486
\end{aligned}
$$

and hence $\rho_{\max }=0.1732$. We have designed a hybrid Kalman filter with a careful tuning of the covariance matrices in such a way to provide the best trade between transient and steady-state behavior. Likewise for the hybrid observer, we have applied the same mode estimator based on (15) with the corresponding state estimate as input.

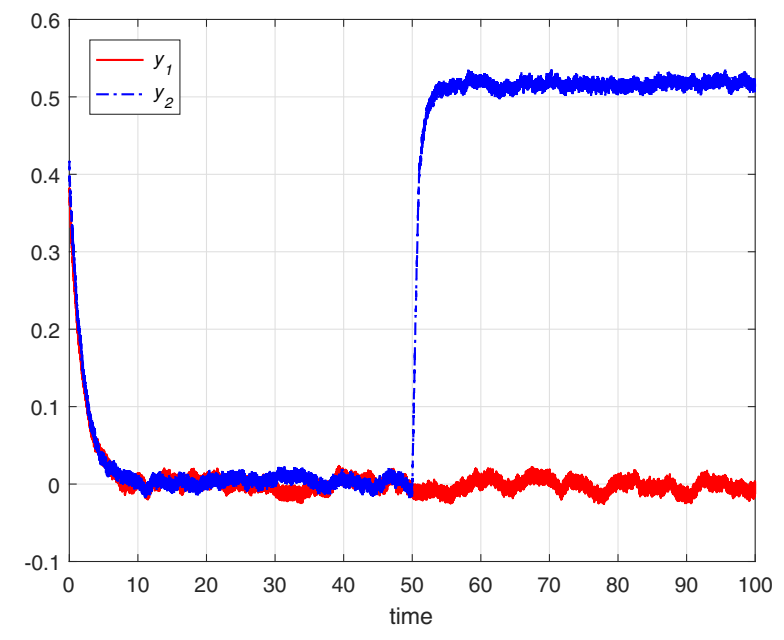

Fig. 1. Time behavior of $y_{1}$ and $y_{2}$ in a simulation run.

\begin{tabular}{ccc}
\hline$\rho$ & KF & QB obs. \\
\hline$\geq 0.20$ & 0 & 0 \\
0.15 & 70.0 & 0 \\
0.10 & 75.7 & 0 \\
0.05 & 100 & 59.6 \\
$\leq 0.01$ & 100 & 100 \\
\hline
\end{tabular}

TABLE II

PERCENTAGE OF FALSE ALARMS FOR KF AND QB OBSERVER OVER 1000 RANDOM RUNS WITH THRESHOLD EQUAL TO $\rho \rho_{\max }$.

Fig.s 1-5 show the result of a simulation run, in which the proposed hybrid observer provides a quick reaction to the occurrence of the fault. To achieve a similar result, the tuning of the hybrid KF turns out to be quite difficult. Depending on the selection of covariance matrix of the measurement

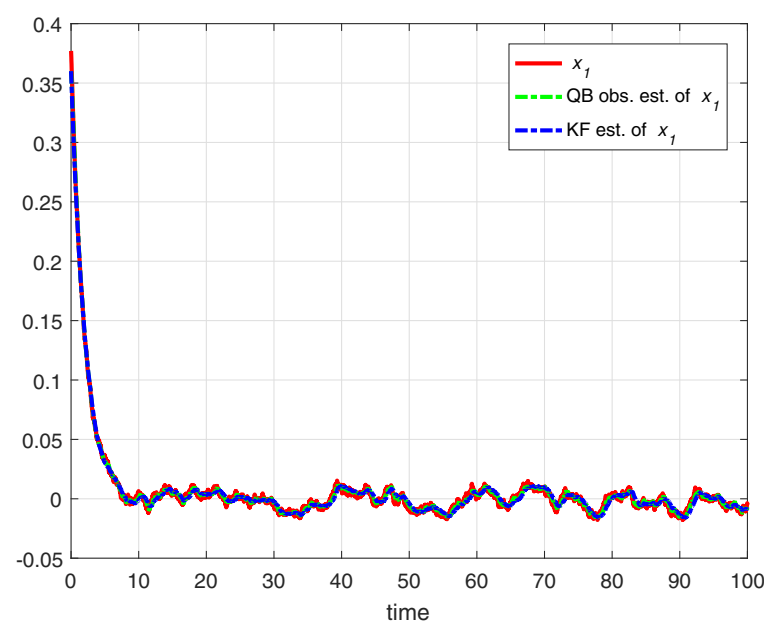

Fig. 2. Time behavior of $x_{1}$ and its estimates in a simulation run.

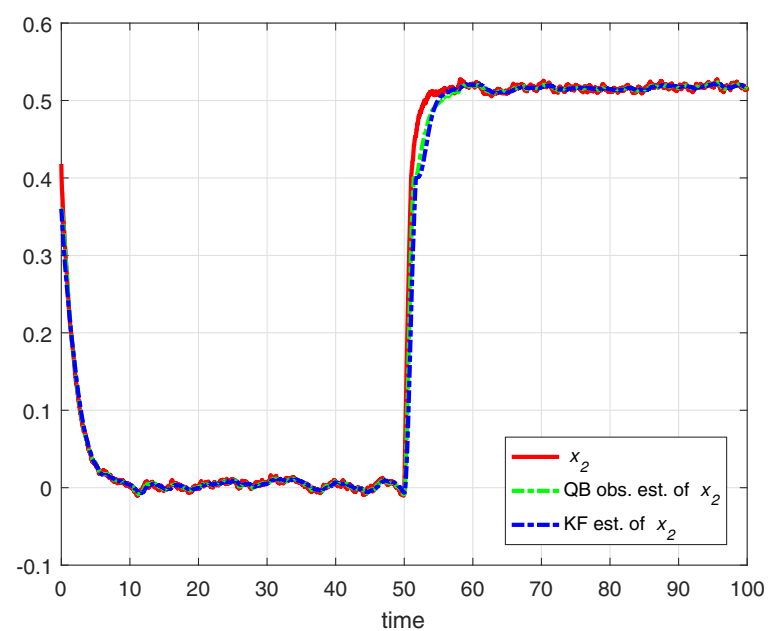

Fig. 3. Time behavior of $x_{2}$ and its estimates in a simulation run.

noises, on one hand the response to the fault may be rapid but with an estimation error very sensitive to the noises or, on the other hand, slow but more robust to noises. Table II shows that the proposed QB approach performs better also in terms of false alarms. In practice, for the proposed approach lower thresholds can be chosen that provide a much smaller false alarm rate as compared with the KF.

\section{CONCLUSIONS}

Fault detection has been addressed by using QB for both LTI and PWA systems. The design of the proposed estimators is accomplished by minimizing a steady-state upper bound in order to make the detection threshold less conservative as much as possible. Such a design is accomplished by means of SDP tools since the QB conditions can be expressed by using LMIs. As a future work we aim at extending our approach to a wider class of plants, especially those usually referred to as distributed systems as well as to nonlinear systems in the presence of non-Gaussian, anomalous noises [32], [33]. 


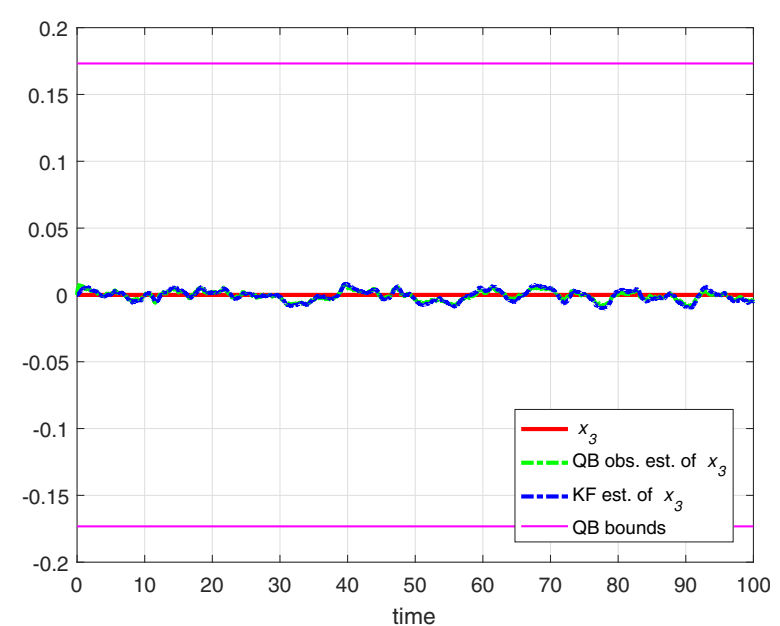

Fig. 4. Time behavior of $x_{3}$ and its estimates in a simulation run.

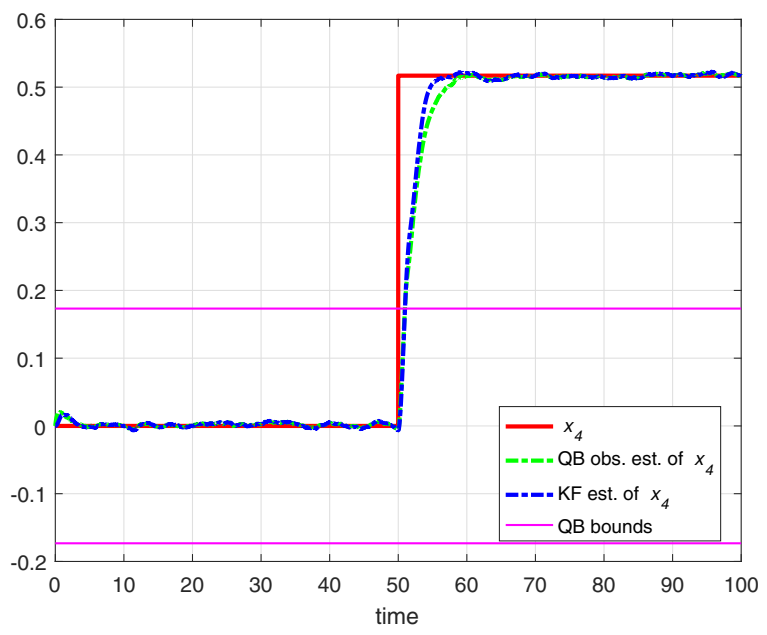

Fig. 5. Time behavior of $x_{4}$ and its estimates in a simulation run.

\section{REFERENCES}

[1] R. Patton, P. Frank, and D. Clark, Fault Diagnosis in Dynamic Systems: Theory and Application. Upper Saddle River, NJ, USA: Prentice Hall, 1989.

[2] M. Blanke, M. Kinnaert, J. Lunze, and M. Staroswiecki, Diagnosis and Fault-Tolerant Control, 2nd ed. Springer Verlag, 2010.

[3] J. Gertler, Fault Detection and Diagnosis. Springer, 2015.

[4] A. Teixeira, I. Shames, H. Sandberg, and K. H. Johansson, "Distributed fault detection and isolation resilient to network model uncertainties," IEEE Trans. Cybernetics, vol. 44, no. 11, pp. 2024-2037, Nov 2014.

[5] M. Staroswiecki and A. Moradi Amani, "Fault-tolerant control of distributed systems by information pattern reconfiguration," Int. Journal of Adaptive Control and Signal Processing, vol. 29, no. 6, pp. 671684, 2015.

[6] M. Blanke, M. Kinnaert, J. Lunze, and M. Staroswiecki, "Distributed fault diagnosis and fault-tolerant control," in Diagnosis and FaultTolerant Control. Springer, 2016, pp. 467-518.

[7] V. Gupta and V. Puig, "Distributed fault diagnosis using minimal structurally over-determined sets: Application to a water distribution network," in 3rd Conference on Control and Fault-Tolerant Systems (SysTol). IEEE, 2016, pp. 811-818.

[8] M. Davoodi, N. Meskin, and K. Khorasani, "Simultaneous fault detection and consensus control design for a network of multi-agent systems," Automatica, vol. 66, pp. 185-194, 2016.

[9] S. Riverso, F. Boem, G. Ferrari-Trecate, and T. Parisini, "Plug-andplay fault detection and control-reconfiguration for a class of nonlinear large-scale constrained systems," IEEE Trans. Automat. Control, vol. 61, no. 12, pp. 3963-3978, 2016.
[10] F. Boem, R. M. Ferrari, C. Keliris, T. Parisini, and M. Polycarpou, "A distributed networked approach for fault detection of large-scale systems," IEEE Trans. Automat. Control, vol. 62, no. 1, pp. 18-33, 2017.

[11] F. Boem, S. Riverso, G. Ferrari-Trecate, and T. Parisini, "Plug-andplay fault detection and isolation for large-scale nonlinear systems with stochastic uncertainties," IEEE Trans. Automat. Control, 2018.

[12] X. Zhang, M. Polycarpou, and T. Parisini, "A robust detection and isolation scheme for abrupt and incipient faults in nonlinear systems," IEEE Trans. Automat. Control, vol. 47, no. 4, pp. 576-593, Apr 2002.

[13] M. Brockman and M. Corless, "Quadratic boundedness of nominally linear systems," Int. Journal of Control, vol. 71, no. 6, pp. 1105-1117, 1998.

[14] S. Olaru, J. A. De Doná, M. M. Seron, and F. Stoican, "Positive invariant sets for fault tolerant multisensor control schemes," Int. Journal of Control, vol. 83, no. 12, pp. 2622-2640, 2010.

[15] F. Xu, V. Puig, C. Ocampo-Martinez, S. Olaru, and F. Stoican, "Settheoretic methods in robust detection and isolation of sensor faults," Int. Journal of Systems Science, vol. 46, no. 13, pp. 2317-2334, 2015.

[16] J. K. Scott, D. M. Raimondo, G. R. Marseglia, and R. D. Braatz, "Constrained zonotopes: A new tool for set-based estimation and fault detection," Automatica, vol. 69, pp. 126-136, 2016.

[17] D. M. Raimondo, F. Boem, A. Gallo, and T. Parisini, "A decentralized fault-tolerant control scheme based on active fault diagnosis," in 2016 IEEE 55th Conference on Decision and Control, 2016, pp. 2164-2169.

[18] B. Ding, "Quadratic boundedness via dynamic output feedback for constrained nonlinear systems in Takagi-Sugeno's form," Automatica, vol. 45, no. 9, pp. 2093 - 2098, 2009.

[19] A. Alessandri, M. Baglietto, and G. Battistelli, "On estimation error bounds for receding-horizon filters using quadratic boundedness," IEEE Trans. Automat. Control, vol. 49, no. 8, pp. 1350-1355, 2004.

[20] A. Alessandri, M. Baglietto, and G. Battistelli, "Design of state estimators for uncertain linear systems using quadratic boundedness," Automatica, vol. 42, no. 3, pp. 497-502, 2006.

[21] J. Lan and R. J. Patton, "A new strategy for integration of fault estimation within fault-tolerant control," Automatica, vol. 69, pp. 4859, 2016.

[22] X. Zhao, H. Liu, J. Zhang, and H. Li, "Multiple-mode observer design for a class of switched linear systems," IEEE Trans. Automation Science and Engineering, vol. 12, no. 1, pp. 272-280, 2015.

[23] R. Ma, J. Fu, and T. Chai, "Dwell-time-based observer design for unknown input switched linear systems without requiring strong detectability of subsystems," IEEE Trans. Automat. Control, vol. 62, no. 8, pp. 4215-4221, 2017.

[24] L. Li, S. X. Ding, J. Qiu, K. Peng, and Y. Yang, "An optimal fault detection approach for piecewise affine systems via diagnostic observers," Automatica, vol. 85, pp. 256 - 263, 2017.

[25] A. Bemporad, G. Ferrari-Treccate, and M. Morari, "Observability and controllability of piecewise affine and hybrid systems," IEEE Trans. Automat. Control, vol. 45, no. 10, pp. 1864-1876, 2000.

[26] A. Alessandri and P. Coletta, "Design of Luenberger observers for a class of hybrid linear systems," in Hybrid Systems: Computation and Control, ser. Lecture Notes in Computer Science, M. D. Di Benedetto and A. Sangiovanni-Vincentelli, Eds. Springer, 2001, pp. 7-18.

[27] A. Alessandri, M. Baglietto, and G. Battistelli, "Luenberger observers for switching discrete-time linear systems," Int. Journal of Control, vol. 80, no. 12, p. 19311943, 2007.

[28] A. Tanwani, H. Shim, and D. Liberzon, "Observability for switched linear systems: Characterization and observer design," IEEE Trans. Automat. Control, vol. 58, no. 4, pp. 891-904, 2013.

[29] S. Boyd, L. El Ghaoui, E. Feron, and V. Balakrishnan, Linear Matrix Inequalities in System and Control Theory, ser. Studies in Applied Mathematics. Philadelphia, PA: SIAM, 1994, vol. 15.

[30] A. Vemuri, "Sensor bias fault diagnosis in a class of nonlinear systems," IEEE Trans. Automat. Control, vol. 46, no. 6, pp. 949-954, 2001.

[31] A. Alessandri and R. Filippini, "Evaluation of resilience of interconnected systems based on stability analysis," in CRITIS 2012, Lecture Notes in Computer Sciences, B. Hämmerli, N. K. Svendsen, and J. Lopez, Eds., vol. 7722. Springer-Verlag, 2013, pp. 180-190.

[32] A. Alessandri and M. Awawdeh, "Moving-horizon estimation with guaranteed robustness for discrete-time linear systems and measurements subject to outliers," Automatica, vol. 67, pp. 85-93, 2016.

[33] A. Alessandri and L. Zaccarian, "Stubborn state observers for linear time-invariant systems," Automatica, vol. 88, pp. 1-9, 2018. 